\title{
Comparison of the antiviral effect of Arbidol and Chloroquine in treating COVID-19
}

\author{
Ming $\mathrm{Li}^{1 \#}$, Tongya Yu ${ }^{1 \#}$, Jinghua Zhu ${ }^{2 \#}$, Yinjia Wang ${ }^{3}$, Yanxia Yang ${ }^{4}$, Kaiwu Zhao ${ }^{5}$, Yongning $\mathbf{Y i}^{6}$, \\ Juan $\mathrm{He}^{7 *}$, Chao $\mathrm{Li}^{4 *}$, Jian $\mathrm{He}^{6 *}$
}

${ }^{1}$ Department of Respiratory and Critical Care Medicine, Shanghai 10th People's Hospital, Shanghai, China; ${ }^{2}$ Department of Surgery, Chibi People's Hospital, Chibi, China; ${ }^{3}$ Department of Intensive Care Unit Medicine, The First People's Hospital of Kunming, Kunming, China; ${ }^{4}$ Department of Respiratory and Critical Care Medicine, The First People's Hospital of Kunming, Kunming, China; ${ }^{5}$ Department of Infectious Disease, The First People's Hospital of Kunming, Kunming, China; ${ }^{6}$ Department of Respiratory and Critical Care Medicine, The 4th Hospital of Kunming City, Anning, China; ${ }^{7} 920$ th Hospital Pharmacy of Joint Logistics Support Force, Kunming, China

Contributions: (I) Conception and design: M Li, J He; (II) Administrative support: M Li, J He; (III) Provision of study materials or patients: J He; (IV) Collection and assembly of data: J He, J Zhu, J He, C Li, Y Wang, Y Yang, K Zhao, Y Yi; (V) Data analysis and interpretation: M Li, T Yu, J Zhu; (VI) Manuscript writing: All authors; (VII) Final approval of manuscript: All authors.

"These authors contributed equally to this work.

*These authors are co-corresponding authors.

Correspondence to: Jian He, MD. Department of Respiratory and Critical Care Medicine, The 4th Hospital of Kunming City, Ganghe South Road, Jinfang Street, Anning 650302, China. Email: ignatias@163.com; Chao Li, MD. Department of Intensive Care Unit Medicine, The First People’s Hospital of Kunming, Qingnian Road, Kunming 650011, China. Email: kmdocli@163.com; Juan He, MD. 920th Hospital Pharmacy of Joint Logistics Support Force, Daguan Road, Kunming 650032, China. Email: hheejuan@126.com.

Background: The novel coronavirus disease 2019 (COVID-19) was broken out in December 2019 and soon became a global health emergency. Effective treatment for COVID-19 is urgently needed. In the present study, we aimed to evaluate the antiviral effect of Arbidol vs. Chloroquine in treating COVID-19.

Methods: We retrospectively analyzed 62 patients with COVID-19 diagnosed according to the guidelines for diagnosis and treatment of COVID-19 in China. They were divided into two groups depending on the antiviral drugs that they received. Participants in the Arbidol group ( $\mathrm{n}=42)$ received $0.2 \mathrm{~g}$ Arbidol, tid for 10 days, and those in Chloroquine group $(\mathrm{n}=20)$ received $500 \mathrm{mg}$ Chloroquine, bid for 10 days. The coronavirus negative conversion time and the length of hospital stay were analyzed and compared between the two groups. Results: There was no significant difference in demographic and clinical characteristics between the two groups. After antiviral treatment, the nasopharyngeal specimen negative conversion time of severe acute respiratory syndrome coronavirus 2 (SARS-CoV-2) and the length of hospital stay in the Arbidol group were significantly shorter than those in the Chloroquine group (18.50 vs. 25.05 days, $\mathrm{P}=0.001 ; 23.52$ vs. 28.75 days, $\mathrm{P}=0.001)$. Adverse events observed during the antiviral treatment period were comparable between the two groups. Overall, 3 (7.14\%) participants in the Arbidol group and 4 (20.0\%) in the Chloroquine group experienced adverse events during antiviral treatment.

Conclusions: These results suggest that Arbidol is advantageous over Chloroquine in terms of the SARS$\mathrm{CoV}-2$ negative conversion and the length of hospital stay in treating COVID-19 patients.

Keywords: COVID-19; Arbidol; Chloroquine; comparative study

Submitted Jan 27, 2021. Accepted for publication Mar 23, 2021.

doi: 10.21037/apm-21-400

View this article at: http://dx.doi.org/10.21037/apm-21-400

$\wedge$ ORCID: 0000-0003-4162-2802. 


\section{Introduction}

The novel coronavirus disease 2019 (COVID-19) broke out at the end of 2019 and soon became a global health emergency. By 20 September 2020, the outbreak of COVID-19 had infected more than 30 million people and caused more than 950 thousand deaths globally. Many articles on the COVID-19 have been published, mainly involving the molecular mechanism, treatment, prevention, and public health management of COVID-19. Treatment of the COVID-19 is mainly related to antiviral and immunotherapy. The present study focused on the antiviral treatment of COVID-19.

On 19 February 2020, the National Health Commission (NHC) of the People's Republic of China issued the sixth edition of the Guidelines for the Prevention, Diagnosis, and Treatment of Novel Coronavirus-induced Pneumonia, which for the first time recommended the use of Arbidol and Chloroquine for the treatment of COVID-19. However, clinical evidence about the efficacy of Arbidol and Chloroquine for the treatment of COVID-19 is currently very limited.

Arbidol is a non-nucleoside membrane fusion inhibitor, which blocks the interaction between the influenza virus and host cells. Evidence has shown that Arbidol also has an antiviral effect against severe acute respiratory syndrome coronavirus (SARS-CoV) $(1,2)$. Chloroquine is an antimalarial drug, which was reported as a potential anti-viral drug based on inhibiting acidification of endosomes (3), and has been shown to block replication of SARS-CoV-2/2019 novel coronavirus (2019-nCoV) in vitro (4).

In this study, 62 patients with COVID-19 were analyzed to determine their clinicopathological characteristics, and evaluate the antiviral effects of Arbidol and Chloroquine and their clinical outcomes in these patients.

We present the following article in accordance with the STROBE reporting checklist (available at http://dx.doi. org/10.21037/apm-21-400).

\section{Methods}

A total of 62 patients who were diagnosed with COVID-19 and received treatment in the Hubei Chibi Hospital (Chibi, China) from January to March 2020 were analyzed retrospectively. All participants had typical clinical symptoms of novel coronavirus pneumonia and pulmonary computed tomography (CT) imaging characteristics, and their nasopharyngeal specimens were positive for SARS-
$\mathrm{CoV}-2$ as detected by reverse transcription polymerase chain reaction (RT-PCR) in two consecutive tests according to the diagnostic criteria of the Chinese guidelines for the diagnosis and treatment of COVID-19. The 62 participants were allocated to two groups based on whether Arbidol or Chloroquine was used as antiviral treatment.

All procedures performed in this study involving human participants were in accordance with the Declaration of Helsinki. The study was approved by Ethics Committee of Chibi People's Hospital of Hubei Province (No.: HBCBHIEC-2020-101). Individual consent for this retrospective analysis was waived.

\section{Procedures}

Antiviral treatment was initiated upon diagnosis of COVID-19. Participants in the Arbidol group ( $n=42)$ received $0.2 \mathrm{~g}$ Arbidol (Simcere, Nanjing, China), 3 times a day (tid) for 10 days, and those in Chloroquine group $(n=20)$ received $500 \mathrm{mg}$ Chloroquine (Wuzhong Pharmceuticals, Suzhou, China), 2 times a day (bid) for 10 days. In addition to the antiviral drugs, all participants received other symptomatic treatments, including anti-bacterial infection therapy, oxygen inhalation, anti-tussives, and expectorant therapy.

\section{Clinical assessments and outcomes}

The primary endpoint was a negative conversion time of SARS-CoV-2 from the date of COVID-19 diagnosis, and the length of hospital stay was also analyzed.

\section{Statistical analysis}

Using the software SPSS version 23.0 (IBM Corp., Armonk, NY, USA), normal distribution measurement data were expressed as $\mathrm{x} \pm \mathrm{s}$. Comparison of the mean values between the two groups was performed by a $t$-test. Non-normal distribution measurement data were described by median using the rank-sum test. Count data were analyzed using the chi-square test. A P value of less than 0.05 was considered statistically significant.

\section{Results}

\section{Demographic and clinical characteristics}

Of the 62 COVID-2019 participants from January to March 
Table 1 Clinical characteristics of the two different treatment groups

\begin{tabular}{|c|c|c|c|}
\hline Characteristics & Chloroquine group $(n=20)$ & Arbidol group $(n=42)$ & $P$ value \\
\hline Gender (male/female) & $7 / 13$ & $17 / 25$ & 0.78 \\
\hline Time of onset of disease & $4.60 \pm 1.18$ & $4.76 \pm 0.98$ & 0.15 \\
\hline Temperature $\left({ }^{\circ} \mathrm{C}\right)$ & $37.4 \pm 0.79$ & $37.0 \pm 0.69$ & 0.13 \\
\hline CRP level (mg/L) & $29.85 \pm 22.33$ & $38.07 \pm 26.67$ & 0.32 \\
\hline Albumin (g/L) & $39.51 \pm 3.06$ & $39.43 \pm 2.89$ & 0.52 \\
\hline Underlying disease (yes/no) & $11 / 9$ & $21 / 21$ & 0.46 \\
\hline Smoking history (yes/no) & $5 / 15$ & $3 / 39$ & 0.09 \\
\hline Consolidation (yes/no) & $4 / 16$ & $9 / 33$ & 1.0 \\
\hline No. lobes affected & $2.30 \pm 0.57$ & $2.17 \pm 0.58$ & 0.23 \\
\hline
\end{tabular}

$\mathrm{N}$, number; CRP, C-reactive protein; CT, computed tomography.

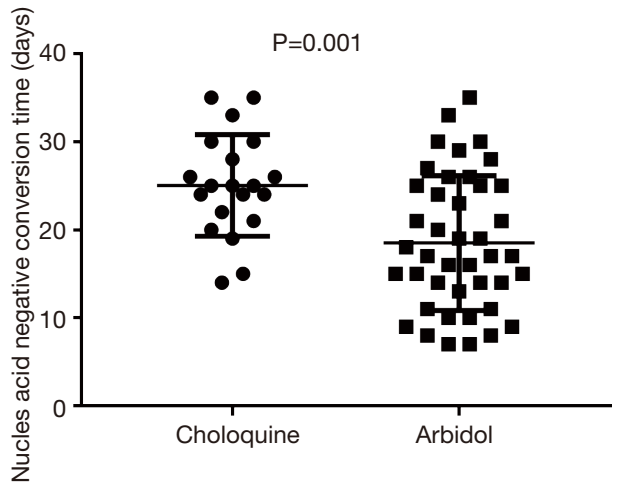

Figure 1 Effect on the time to conversion to nucleic acid negativity.

2020, 42 received Arbidol and 20 received Chloroquine treatment. Comparisons of the baseline characteristics between the two groups are presented in Table 1. There was no significant difference in patient age, gender, time of disease onset, body temperature, oxygen saturation, C reactive protein (CRP) level, albumin, smoking history, and CT imaging characteristics between the two groups (all $\mathrm{P}>0.05$ ) (Table 1).

\section{Nasopharyngeal specimens negative conversion of SARS- $\mathrm{CoV}-2$}

After 7 days of treatment, negative conversion of SARS$\mathrm{CoV}-2$ was detected in $2(4.76 \%)$ of the 42 nasopharyngeal specimens in the Arbidol group, and no negative conversion was detected in the Chloroquine group. After 14 days of treatment, negative conversion of SARS-CoV-2 was detected in $12(28.57 \%)$ of the 42 nasopharyngeal specimens in the Arbidol group, and in $1(5.00 \%)$ of the 20 nasopharyngeal specimens in the Chloroquine group. The mean negative conversion time of the 62 participants with COVID-19 was 20.62 days (18.50 days in Arbidol group and 25.05 days in Chloroquine group) showing a significant difference between the two groups $(\mathrm{P}=0.001)$. These results suggest that Arbidol offered a shorter nasopharyngeal specimen negative conversion time of SARS-CoV-2 compared with Chloroquine (Figure 1).

\section{Length of hospital stay}

The mean length of hospital stay of the 62 participants was 25.20 days (23.52 days in Arbidol group and 28.75 days in 


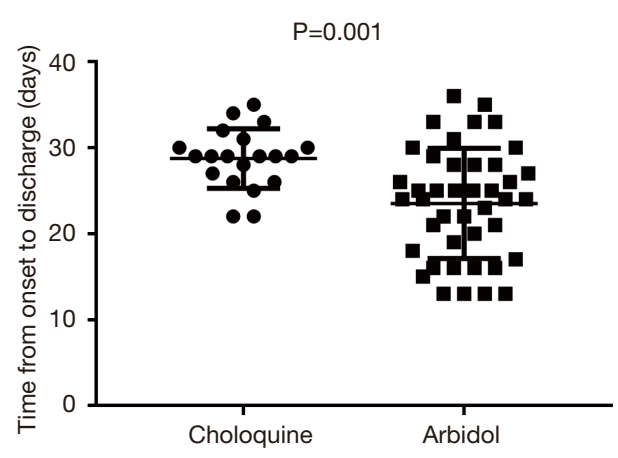

Figure 2 Effect on length of hospital stay.

Chloroquine group), and the difference between the two groups was significant $(\mathrm{P}=0.001)$. The shortest length of hospital stay in the Arbidol group was 13 days, including $4(9.52 \%)$ of the 42 participants who were discharged from hospital within 14 days. In Chloroquine group, the shortest length of hospital stay was 22 days, and nobody was discharged from hospital within 14 days. These results suggest that Arbidol had an advantage over Chloroquine in terms of hospitalization time (Figure 2).

\section{Adverse events during the antiviral treatment period}

The most common adverse events during antiviral treatment with Arbidol and Chloroquine included vomiting and hepatic function impairment. A participant in the Arbidol group and two participants in the Chloroquine group experienced vomiting. The alanine aminotransferase (ALT) level was slightly elevated in two patients in both the Arbidol and Chloroquine groups. Overall, all participants in both groups tolerated the treatment well.

\section{Discussion}

Since the COVID-19 epidemic broke out globally, great progress has been made in the prevention and management of covid-19. In China, COVID-19 is effectively suppressed by critical policy decisions made by the government and public health institutions. Effective measures include reducing unnecessary personnel flow, rapidly screening and isolating potential patients with COVID-19, and popularizing nucleic acid testing. In addition, the rapid development and vaccination of the covid-19 vaccine has also effectively limited the prevalence of the disease. Since the COVID-19 does not have certain remedy, treatment relies mainly on existing drugs, especially antiviral drugs.
After being approved by the Chinese National Health Commission, Arbidol and Chloroquine have been tentatively used in the clinical treatment of COVID- 19 . In the present study, we analyzed 62 COVID-19 patients to compare the efficacy of Arbidol and Chloroquine in the treatment of COVID-19 using nasopharyngeal specimen negative conversion time as the primary endpoint to evaluate their antiviral effects, respectively. The resulting data showed that the mean nasopharyngeal specimen negative conversion time in the Arbidol group was shorter than that in the Chloroquine group, and the length of hospital stay in the Arbidol group was also shorter. These results indicated that Arbidol is advantageous over Chloroquine in treating COVID-19.

So far, there have been limited prospective randomized controlled trials (RCTs) reporting potentially effective antiSARS-CoV-2 therapies, and the published data on the effect of Arbidol in treating COVID-19 are controversial. Zhu et al. (5) retrospectively analyzed 50 COVID-19 patients in a lopinavir/ritonavir (LPV/r) group (n=34) and an Arbidol group $(\mathrm{n}=16)$, and found that the SARS-CoV-2 nucleic acid negative conversion rate in the Arbidol group was higher than that in LPV/r group at 7 and 14 days after treatment, indicating that Arbidol monotherapy is superior to LPV/r in treating COVID-19. However, in an exploratory RCT, Li et al. (6) observed 86 patients with mild/moderate COVID-19, including 34 in a LPV/r group, 35 in an Arbidol group, and 17 in the control group (receiving no antiviral treatment). They reported that patients in the Arbidol monotherapy group did not gain significant benefits compared with the LPV/r or control groups in terms of positive-to-negative conversion of SARS-CoV-2 nucleic acid or symptomatic alleviation. The difference may be attributable to the small sample size of both studies and severity of pneumonia. In addition, patient underlying diseases and general conditions may also have contributed to the difference. In Zhu's study, patients in the Arbidol group were younger than those in LPV/r group, which may have led to more obvious advantages in the Arbidol group.

In addition to Arbidol monotherapy, some studies have explored the combination of Arbidol with other therapies. $\mathrm{Xu}$ et al. (7) reported that combined use of interferon a2b (IFN-a2b) and Arbidol was superior to IFN$\mathrm{a} 2 \mathrm{~b}$ monotherapy in absorption of pneumonia, but had no significant advantage in duration of viral RNA in the respiratory tract and length of hospital stay. Deng et al. (8) reported that combined use of Arbidol and LPV/r was superior to LPV/r monotherapy in shortening SARS-CoV-2 
negative conversion time and pneumonia absorption. These studies suggest that Arbidol may be effective in treating COVID-19, and combined application of adjuvant therapy seems to be more beneficial.

Existing evidence shows that Arbidol plays a role not only in treatment but also in the prevention of COVID-19. Zhang's study (9) showed that post-exposure prophylaxis using Arbidol was a protective factor against the development of COVID-19 and was associated with decreased infection among family members and health care workers exposed to confirmed cases of COVID-19 infection. Yang et al. (10) reported that prophylactic oral administration of Arbidol among frontline healthcare workers could lower their infection rate.

Besides Arbidol, Chloroquine is also a drug recommended by many clinicians. Huang et al. (11) conducted a retrospective cohort study in 27 patients with COVID-19 patients, among whom 10 patients received Chloroquine, 11 patients received Arbidol, and 6 patients received LPV/r. The results showed that both Arbidol and Chloroquine were effective in shortening the viral shedding interval and the length of hospital stay, and reducing hospitalization expenses compared with LPV/r group, but there was no statistically significant difference between Chloroquine and Arbidol groups. It was found in our study that Arbidol treatment shortened the nucleic acid negative conversion time and the length of hospital stay as compared with Chloroquine. The difference may be attributable to the small sample size of both studies. In the absence of a consensus treatment, Arbidol seems to be a preferable remedy for the clinical treatment of COVID-19.

The main concern about antiviral treatment with Arbidol and Chloroquine is their adverse effects (12-14). Chloroquine has been used to treat malaria and autoimmune diseases, and has adverse effects that are well known and can be severe, including psychiatric effects, cardiac toxicity, arrhythmia, and sudden death (15). The reported adverse effects of Arbidol mainly include nausea, diarrhea, dizziness, and elevated serum transaminase. In this study, vomiting and elevation of ALT appeared in both the Arbidol and Chloroquine groups, and no other serious adverse effects were observed. For antiviral treatment of COVID-19, Arbidol and Chloroquine are relatively safe when administered at their respective recommended dosages $(5,16)$.

In the present study, due to the small sample size, it is difficult to summarize convincing clinical features of COVID-19 patients with longer negative conversion time.
And existing studies have shown that in the elderly, male, later hospitalization time or later antiviral treatment, mechanical ventilation delays in clearance of SARS-CoV-2 RNA (17). Besides patients with coronary heart disease or hypoalbuminemia have longer virus positive time (18). These studies tell us that more attention should be paid to patients with these characteristics, and treatment plan should be adjusted for these patients.

The present study enrolled more patients and drew more robust conclusions on the nucleic acid negative conversion time, the length of hospital stay and related complications. However, there are several limitations in the present study including its retrospective nature and the study being performed in a single hospital, which might have the potential of selection bias, though we included all COVID-19 patients who have received Chloroquine and Arbidol in the Hubei Chibi Hospital (Chibi, China) from January to March 2020 and meet the inclusion criteria. Further randomized controlled trials with a much larger sample size are needed to find effective treatment.

In conclusion, our study demonstrated that Arbidol monotherapy was advantageous in treating COVID-19 in terms of shortening the nucleic acid negative conversion time and the length of hospital stay. But as the sample size of this study was relatively small, RCTs with larger-sample sizes are required to verify this conclusion.

\section{Acknowledgments}

Funding: This work was financially supported by the Fundamental Research Funds for the Central Universities (22120180584), Shanghai Tenth Hospital's Improvement Plan for NSFC (04.03.17.032, 04.01.18.048, SYGZRPY2017014) and Scientific Research Projects of Shanghai Municipal Commission of Health and Family Planning (201840056).

\section{Footnote}

Reporting Checklist: The authors have completed the STROBE reporting checklist. Available at http://dx.doi. org/10.21037/apm-21-400

Data Sharing Statement: Available at http://dx.doi. org/10.21037/apm-21-400

Conflicts of Interest: All authors have completed the ICMJE uniform disclosure form (available at http://dx.doi. 
org/10.21037/apm-21-400). The authors have no conflicts of interest to declare.

Ethical Statement: The authors are accountable for all aspects of the work in ensuring that questions related to the accuracy or integrity of any part of the work are appropriately investigated and resolved. All procedures performed in this study involving human participants were in accordance with the Declaration of Helsinki (as revised in 2013). The study was approved by Ethics Committee of Chibi People's Hospital of Hubei Province (No.: HBCBHIEC-2020-101). Individual consent for this retrospective analysis was waived.

Open Access Statement: This is an Open Access article distributed in accordance with the Creative Commons Attribution-NonCommercial-NoDerivs 4.0 International License (CC BY-NC-ND 4.0), which permits the noncommercial replication and distribution of the article with the strict proviso that no changes or edits are made and the original work is properly cited (including links to both the formal publication through the relevant DOI and the license). See: https://creativecommons.org/licenses/by-nc-nd/4.0/.

\section{References}

1. Choudhary S, Silakari O. Scaffold morphing of arbidol (umifenovir) in search of multi-targeting therapy halting the interaction of SARS-CoV-2 with ACE2 and other proteases involved in COVID-19. Virus Res 2020;289:198146.

2. Barnard DL, Kumaki Y. Recent developments in anti-severe acute respiratory syndrome coronavirus chemotherapy. Future Virol 2011;6:615-31.

3. Yan Y, Zou Z, Sun Y, et al. Anti-malaria drug chloroquine is highly effective in treating avian influenza A H5N1 virus infection in an animal model. Cell Res 2013;23:300-2.

4. Wang $M$, Cao R, Zhang L, et al. Remdesivir and chloroquine effectively inhibit the recently emerged novel coronavirus (2019-nCoV) in vitro. Cell Res 2020;30:269-71.

5. Zhu Z, Liu Z, Xu T, et al. Arbidol monotherapy is superior to lopinavir/ritonavir in treating COVID-19. J Infect 2020;81:e21-e23.

6. Li Y, Xie Z, Lin W, et al. Efficacy and Safety of Lopinavir/ Ritonavir or Arbidol in Adult Patients with Mild/Moderate COVID-19: An Exploratory Randomized Controlled Trial. Med (N Y) 2020;1:105-113.e4.
7. Xu P, Huang J, Fan Z, et al. Arbidol/IFN-a2b therapy for patients with corona virus disease 2019: a retrospective multicenter cohort study. Microbes Infect 2020;22:200-5.

8. Deng L, Li C, Zeng Q, et al. Arbidol combined with LPV/ r versus LPV/r alone against Corona Virus Disease 2019: A retrospective cohort study. J Infect 2020;81:e1-e5.

9. Zhang JN, Wang WJ, Peng B, et al. Potential of Arbidol for Post-exposure Prophylaxis of COVID-19 Transmission: A Preliminary Report of a Retrospective Cohort Study. Curr Med Sci 2020;40:480-5.

10. Yang C, Ke C, Yue D, et al. Effectiveness of Arbidol for COVID-19 Prevention in Health Professionals. Frontiers in Public Health 2020;8:249.

11. Huang H, Guan L, Yang Y, et al. Chloroquine, arbidol (umifenovir) or lopinavir/ritonavir as the antiviral monotherapy for COVID-19 patients: a retrospective cohort study. Research Square 2020;1-15.

12. Zhang XL, Li ZM, Ye JT, et al. Pharmacological and cardiovascular perspectives on the treatment of COVID-19 with chloroquine derivatives. Acta Pharmacol Sin 2020;41:1377-86.

13. Moore N. Chloroquine for COVID-19 Infection. Drug Saf 2020;43:393-4.

14. Yao X, Ye F, Zhang M, et al. Could Chloroquine / Hydroxychloroquine Be Harmful in Coronavirus Disease 2019 (COVID-19) Treatment? Clin Infect Dis 2020;71:732-9.

15. Plantone D, Koudriavtseva T. Current and Future Use of Chloroquine and Hydroxychloroquine in Infectious, Immune, Neoplastic, and Neurological Diseases: A MiniReview. Clin Drug Investig 2018;38:653-71.

16. Huang $M$, Tang T, Pang P, et al. Treating COVID-19 with Chloroquine. J Mol Cell Biol 2020;12:322-5.

17. Xu K, Chen Y, Yuan J. Factors associated with prolonged viral RNA shedding in patients with COVID-19. Clin Infect Dis 2020;71:799-806.

18. Fu Y, Han $P, Z$ Zhu R, et al. Risk factors for viral RNA shedding in COVID-19 patients. Eur Respir J 2020;56:2-4.

(English Language Editor: J. Jones)

Cite this article as: Li M, Yu T, Zhu J, Wang Y, Yang Y, Zhao $\mathrm{K}, \mathrm{Yi} \mathrm{Y}, \mathrm{He} \mathrm{J}, \mathrm{Li} \mathrm{C}, \mathrm{He}$ J. Comparison of the antiviral effect of Arbidol and Chloroquine in treating COVID-19. Ann Palliat Med 2021;10(3):3307-3312. doi: 10.21037/apm-21-400 